\title{
High prevalence of acute myocardial damage in a hospital necropsy series, shown by C9 immunohistology
}

\author{
M A S Ferreira, H E Owen, A J Howie
}

\begin{abstract}
Aims-To use a sensitive test of acute myocardial damage-immunohistological detection of complement component C9-to assess the prevalence of damage in an unselected series of hearts taken at necropsy in adults.

Methods-Sections of formalin fixed and paraffin wax embedded myocardium were cut from 128 consecutive necropsy cases on which a block of heart had been taken. These were stained with an immunohistological method for C9. Necropsy findings were reviewed and clinical risk factors for myocardial damage noted. The extent of C9 immunostaining was correlated with clinical and pathological findings.

Results-There was immunostaining for C9 in 109 heart sections $(85 \%)$. Most had conventional evidence of coronary artery disease or acute or chronic myocardial abnormality, but necrosis was identified by orthodox microscopy in only $12(11 \%$ of C9 positive cases). In 29 cases, orthodox
\end{abstract}

Department of Pathology, University of Birmingham, Birmingham, UK M A S Ferreira H E Owen A J Howie

Correspondence to: Dr A J Howie, Department of Pathology, The Medical School, Birmingham B15 2TT, UK; email

a.j.howie@bham.ac.uk

Accepted for publication 30 March 1998

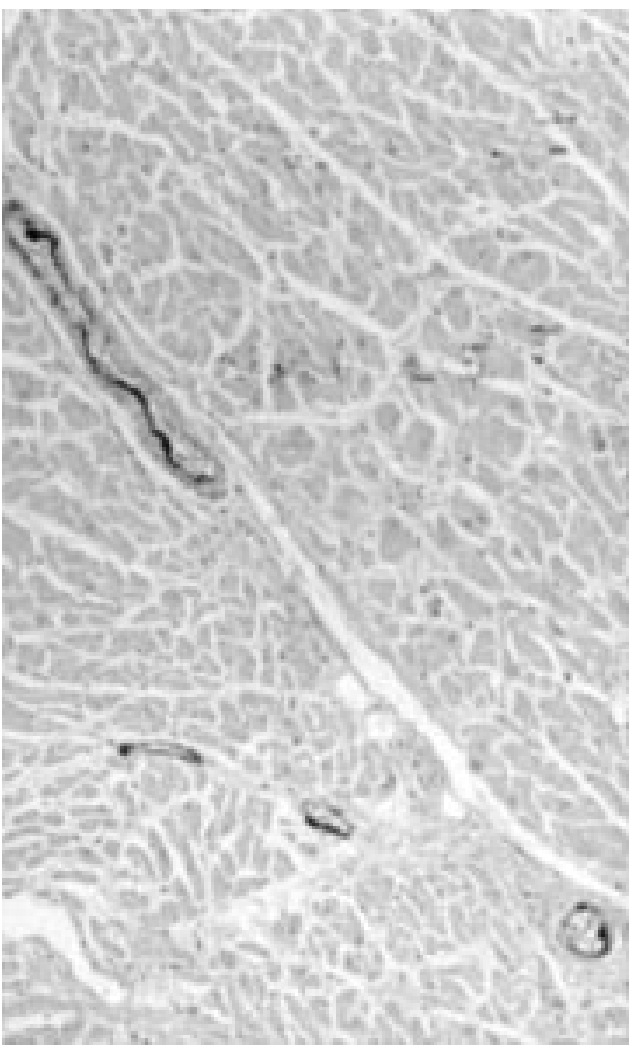

Figure 1 Necropsy heart examined by the immunohistological method for C9. Only the walls of small blood vessels are stained. Negative (grade 0) C9 staining. examination showed no abnormality, but C9 was detected. These cases had clinical risk factors for damage such as hypoxia and hypotension. Increasing age, heart weight, and total number of risk factors and pathological findings were associated with increasing extent of C9 immunostaining.

Conclusions-Acute myocardial damage was common in a hospital necropsy series and its prevalence was underestimated by conventional pathological techniques. Immunostaining for C9 was a simple and useful way of detecting such damage. (F Clin Pathol 1998;51:548-551)

Keywords: complement component C9; heart; myocardial damage

Assessment of acute myocardial damage at necropsy can be difficult. Early myocardial infarction or diffuse damage can be missed with conventional methods of examination of the

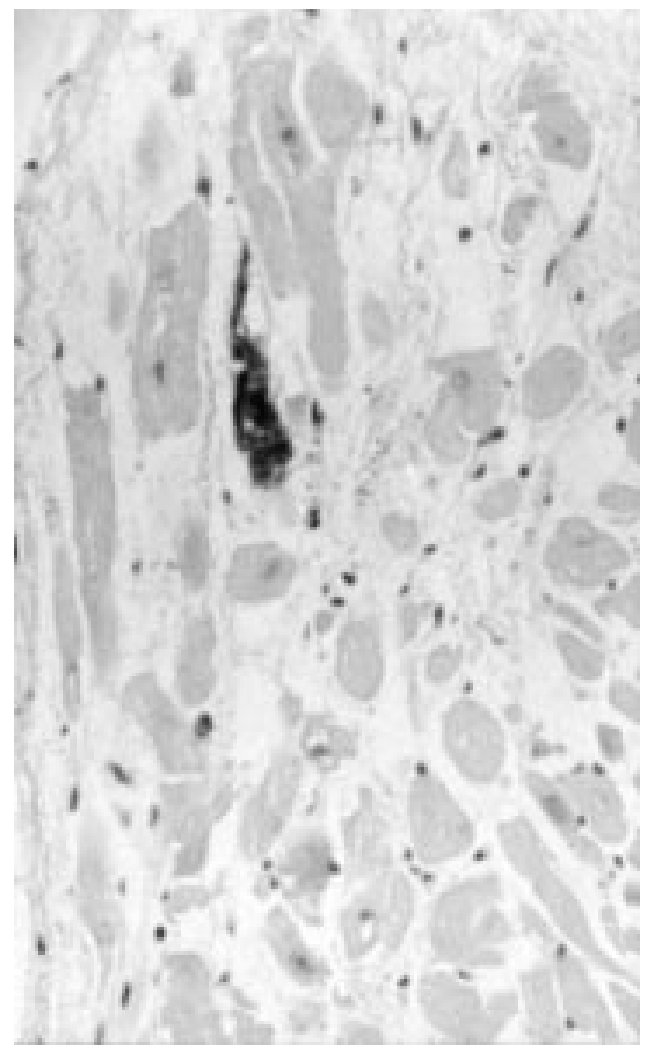

Figure 2 Necropsy heart with a single muscle cell detected by the immunohistological method for C9. Grade $1+C 9$ staining. 


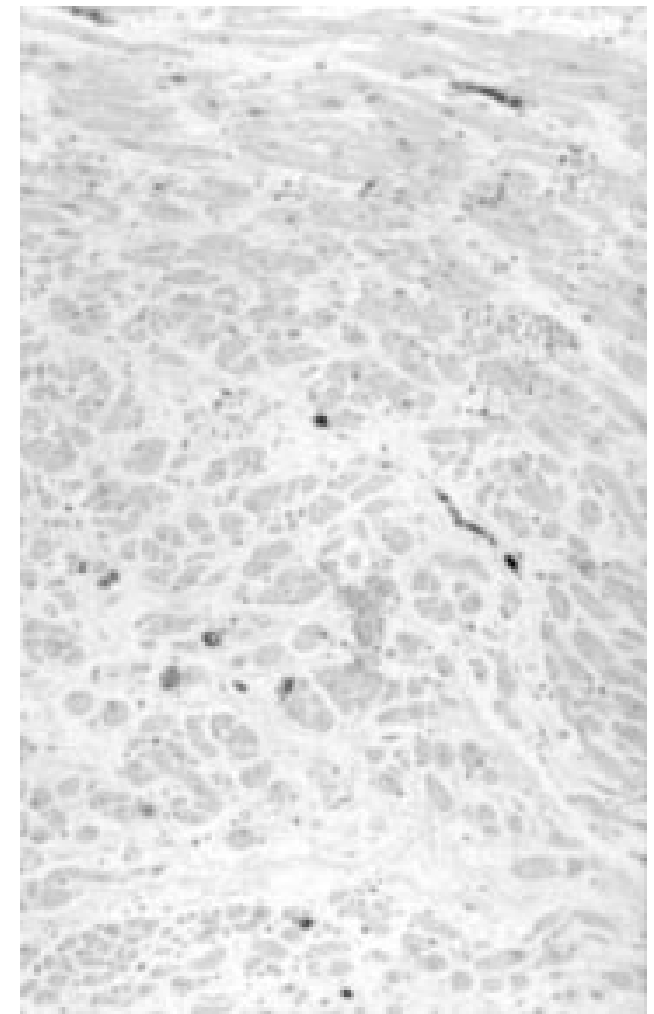

Figure 3 Necropsy heart with a few single cells and small groups of cells detected by the immunohistological method for C9. Grade 2+C9 staining.

heart, and techniques such as enzyme histochemistry are usually considered of little practical value.

The terminal C5b-9 membrane attack complex of the complement system is deposited in necrotic skeletal muscle ${ }^{1}$ and necrotic myocardium..$^{2-5}$ An immunohistological method of detection of deposition of the complement component C9 was found to be a useful way of showing myocardial necrosis in routine necropsy practice in a study validating it against an established method-the nitroblue tetrazolium/ phenazine methosulphate reaction. ${ }^{6}$

We wished to see how commonly the C9 immunostaining method would indicate acute myocardial damage in a consecutive series of necropsies, and to compare the findings with those of orthodox pathological investigation of the heart at necropsy.

\section{Methods}

Cases were studied retrospectively. They were 128 consecutive necropsies on adults at the Queen Elizabeth Hospital, Birmingham, on which a block of left ventricular myocardium was available, fixed in formol saline and embedded in paraffin wax. Some cases, such as heart allografts, had several blocks available but for the purposes of this study only one was taken. Twelve cases had two different sections of heart on the same block. Sections were cut at $5 \mu \mathrm{m}$ thickness and stained by an indirect immunoperoxidase method as before. ${ }^{6}$ In brief, dewaxed sections were treated with hydrogen peroxide in methanol to block endogenous peroxidase, incubated with sheep antiserum to C9 (The Binding Site, Birmingham, UK) at

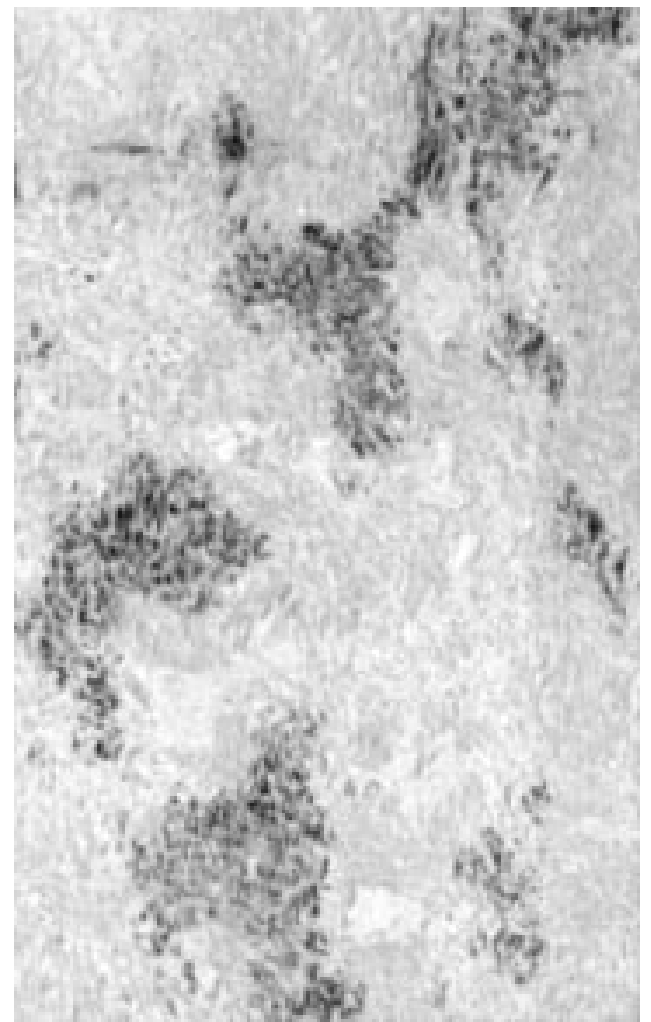

Figure 4 Necropsy heart with large groups of cells detected by the immunohistological method for C9. Grade 3+C9 staining.

1:300 on a Sequenza immunostaining centre (Shandon, Basingstoke, Hampshire, UK), incubated with rabbit anti-sheep immunoglobulins, peroxidase conjugated, at 1:100, covered with hydrogen peroxide and tetra-aminobiphenyl hydrochloride (diaminobenzidine), and counterstained with haematoxylin. With each batch of sections, one section was included of a myocardial infarct known to stain strongly with the antibody to $\mathrm{C} 9$, and many sections contained their own positive control since walls of small blood vessels usually reacted with the antibody to $\mathrm{C} 9$.

The extent of C9 immunostaining was assessed on each section without knowledge of clinical or pathological findings, and was divided into four groups: negative when no myocardial cells were stained; $1+$ when a few single myocardial cells were stained; $2+$ when groups of myocardial cells were stained; and 3+ when a large area of staining was found (figs $1-4)$.

Necropsy reports were analysed for evidence from the macroscopic findings of myocardial abnormality or coronary artery disease. Haematoxylin and eosin sections were reviewed. Clinical risk factors for myocardial damage were also noted. Pathological and clinical features are listed in table 1 . The definition of cardiac enlargement is arbitrary and the weights used in table 1 to define cardiomegaly ${ }^{7}$ were to allow for the fact that pathologists had different assessments of this. C9 immunostaining was then correlated with the risk factors and the pathological findings. 
Table 1 Definitions of clinical risk factors and pathological findings

Clinical risk factors for myocardial damage

Hypotension including hypovolaemia

Systemic or pulmonary hypertension

Hypoxia with or without ventilation

Chronic renal failure and/or dialysis for acute or chronic renal failure

Septicaemia

Diabetes mellitus

Major surgery

Chemotherapy

Severe anaemia

Heart transplantation

Conventional evidence of myocardial abnormality or coronary artery disease

Major coronary artery atherosclerosis with/without thrombosis, only noted in the absence of any other feature in this section

Recent myocardial infarct

Myocardial fibrosis including previous myocardial infarct

Heart weight at least $450 \mathrm{~g}$ (female), $500 \mathrm{~g}$ (male)

Myocarditis and myocardial abscess

Calcification of myocardium

Heart allograft

Table 2 C9 immunostaining related to pathological findings, with sex distribution, mean age $\dagger$, and mean heart weight $\ddagger$ of groups listed in table 3

\begin{tabular}{|c|c|c|c|}
\hline C9 immunostaining & $\begin{array}{l}\text { No conventional evidence of } \\
\text { myocardial damage or } \\
\text { coronary artery disease }\end{array}$ & $\begin{array}{l}\text { Conventional evidence of } \\
\text { myocardial damage or } \\
\text { coronary artery disease }\end{array}$ & Overall \\
\hline$\overline{0}$ & $\begin{array}{l}\text { Group A: } 11(7 \mathrm{f}, 4 \mathrm{~m}) \\
\dagger 49 \text { years } \\
\ddagger 349 \mathrm{~g}\end{array}$ & $\begin{array}{l}\text { Group D: } 8(3 \mathrm{f}, 5 \mathrm{~m}) \\
66 \text { years } \\
422 \mathrm{~g}\end{array}$ & $\begin{array}{l}19(10 \mathrm{f}, 9 \mathrm{~m}) \\
56 \text { years } \\
380 \mathrm{~g}\end{array}$ \\
\hline $1+$ & $\begin{array}{l}\text { Group B: } 16(9 \mathrm{f}, 7 \mathrm{~m}) \\
62 \text { years } \\
356 \mathrm{~g}\end{array}$ & $\begin{array}{l}\text { Group E: } 20(11 \mathrm{f}, 9 \mathrm{~m}) \\
61 \text { years } \\
451 \mathrm{~g}\end{array}$ & $\begin{array}{l}36(20 \mathrm{f}, 16 \mathrm{~m}) \\
61 \text { years } \\
410 \mathrm{~g}\end{array}$ \\
\hline $2+$ & $\begin{array}{l}\text { Group C: } 13(6 \mathrm{f}, 7 \mathrm{~m}) \\
60 \text { years } \\
365 \mathrm{~g}\end{array}$ & $\begin{array}{l}\text { Group F: } 35(17 \mathrm{f}, 18 \mathrm{~m}) \\
72 \text { years } \\
463 \mathrm{~g}\end{array}$ & $\begin{array}{l}48(23 \mathrm{f}, 25 \mathrm{~m}) \\
69 \text { years } \\
438 \mathrm{~g}\end{array}$ \\
\hline $3+$ & None & $\begin{array}{l}\text { Group G: } 25 \text { (9f, } 16 \mathrm{~m}) \\
71 \text { years } \\
484 \mathrm{~g}\end{array}$ & $\begin{array}{l}25(9 \mathrm{f}, 16 \mathrm{~m}) \\
71 \text { years } \\
484 \mathrm{~g}\end{array}$ \\
\hline
\end{tabular}

f, female; m, male.

\section{Results}

The results are summarised in table 2, with details of each patient in table 3. Most hearts$69 \%$ (88 of 128) - had conventional evidence of myocardial abnormality or coronary artery disease. These hearts were heavier than the others (mean weight $462 \mathrm{~g} v 356 \mathrm{~g}$ ) and were from older patients (mean age 69 years $v 58$ years).

Overall, the percentage of cases found positive for C9 was $85 \%$ (109 of 128), with a higher percentage in those with conventional evidence of abnormality ( $91 \% ; 80$ of 88$)$ than in those without $(73 \% ; 29$ of 40$)$. Table 2 shows that as the grade of C9 positivity increased, the mean age increased, the mean heart weight increased, and the relative ratio of those with conventional evidence of abnormality to those without such evidence increasedfrom 0.7 (8:11) in the C9 negative group, through $1.3(20: 16)$ in the group with $1+\mathrm{C} 9$ staining and $2.7(35: 13)$ in the $2+$ group, to infinity $(25: 0)$ in the $3+$ group. This distribution is unlikely to have occurred by chance $\left(\chi^{2}\right.$ $=20.9, \mathrm{p}<0.001$ ).

Of the 109 cases with any grade of C 9 positivity, 29 had no conventional pathological evidence of abnormality (groups B and C). All these had at least one of the clinical risk factors in table 1 . If each clinical risk factor and pathological finding in the heart defined in table 1 is scored 1 in table 3, there is a trend of increasing total scores from groups A and D with negative $\mathrm{C} 9$ staining (mean score 1.8 ), through groups $\mathrm{B}$ and $\mathrm{E}$ with $1+\mathrm{C} 9$ staining (mean score 2.2), to groups $\mathrm{C}$ and $\mathrm{F}$ with $2+\mathrm{C} 9$ staining (mean score 2.7 ) and group $\mathrm{G}$ with $3+$ C9 staining (mean score also 2.7).

Table 3 Details of each group in table 2: clinical risk factors in normal type; pathological findings in bold

No conventional evidence of myocardial damage or coronary artery disease

Group A: C9 immunostaining $0, n=11 \quad$ Hypoxia 4, hypotension 2, hypertension 2, renal failure 1, septicaemia 1, hypoxia + surgery + septicaemia

Group G: C9 immunostaining $1+, \mathrm{n}=16$ Hypoxia 3, hypotension 2, anaemia 2, hypoxia + hypotension 2, hypoxia + renal failure 2 , diabetes 1 , chemotherapy 1 , hypoxia + hypertension 1 , hypotension + anaemia 1 , hypertension + chemotherapy 1

Group C: C9 immunostaining 2+, $\mathrm{n}=13$ Hypoxia 3, hypotension 2, hypotension + anaemia 2, hypertension 1, hypotension + surgery 1 , hypoxia + diabetes + renal failure 1 , hypoxia + surgery + chemotherapy 1 , hypotension + surgery + septicaemia 1 , septicaemia + chemotherapy 1

Conventional evidence of myocardial damage or coronary artery disease

Group D: C9 immunostaining $0, \mathrm{n}=8 \quad$ Hypoxia 3, hypoxia + hypotension 2, hypertension + surgery 1 , diabetes + renal failure 1 , heart transplant 1 ; fibrosis 5 , fibrosis + cardiomegaly 2 , heart transplant 1

Group E: C9 immunostaining 1+, $\mathrm{n}=20$ Hypoxia 6, hypoxia + hypotension 2, hypoxia + hypertension 2, hypotension 1, renal failure 1 , surgery 1 , chemotherapy 1 , heart transplant 1 , hypoxia + anaemia 1 , hypoxia + septicaemia 1 , hypotension + anaemia 1 , hypertension + surgery 1 , hypoxia + renal failure + surgery 1 ; fibrosis 8 , cardiomegaly 5 , fibrosis + cardiomegaly 4 , myocarditis 1 , fibrosis + myocarditis 1 , rejection of transplanted heart 1

Group F: C9 immunostaining 2+, $\mathrm{n}=35$ Hypoxia 11, hypoxia + anaemia 3, hypoxia + hypotension 2, hypoxia + septicaemia 2 , hypotension 1 , hypertension 1 , renal failure 1 , surgery 1 , heart transplant 1 , heart + lung transplant 1 , hypoxia + hypertension 1 , hypoxia + surgery 1 , hypotension + diabetes 1 , hypertension + renal failure 1 , hypertension + anaemia 1 , renal failure + surgery 1 , hypoxia + hypotension + diabetes 1 , hypoxia + renal failure + surgery 1 , hypotension + renal failure + septicaemia 1 , diabetes + anaemia + chemotherapy 1 , hypertension + diabetes + renal failure + septicaemia 1 ; fibrosis 18 , fibrosis + cardiomegaly 10 , cardiomegaly 3 , acute infarct 1 , rejection of transplanted heart 1 , primary failure of transplanted lungs and heart 1 , fibrosis + myocarditis 1

Group G: C9 immunostaining 3+, $\mathrm{n}=25$ Hypoxia 5, hypoxia + hypotension 4, heart transplant 3, septicaemia 2, hypotension 1 , hypertension 1 , renal failure 1 , hypoxia + diabetes 1 , hypotension + diabetes 1 , hypotension + renal failure 1 , hypotension + surgery 1 , hypoxia + hypotension + surgery 1 , hypotension + diabetes + anaemia 1 , no identified factors 2 ; fibrosis 7 , fibrosis + cardiomegaly 5 , acute infarct 3 , cardiomegaly 2 , cardiomegaly + acute infarct 2 , chronic rejection of transplanted heart 2 , calcification 1 , abscess 1 , coronary atherosclerosis 1 , failure of transplanted heart 1 
Myocardial necrosis was seen by conventional microscopy in only 12 cases, 11 in group $\mathrm{G}$ and one in group $\mathrm{F}$ (table 3 ).

\section{Discussion}

Immunohistological demonstration of C9 deposition in the myocardium was shown in an earlier study to be a practical and sensitive method of detecting recent damage, capable of showing necrosis of single myocardial cells. ${ }^{6}$ The method was validated by comparison with an established enzyme histochemical technique, the nitroblue tetrazolium/phenazine methosulphate macroreaction, ${ }^{8}$ and the C9 method was shown to be much easier, much more sensitive, and much more useful in everyday practice.

In the present study, we applied the C9 method retrospectively to a consecutive series of hospital necropsy cases, selected only by the availability of a histological block of left ventricle. This meant that the technique of necropsy examination, the necropsy report, and the part of the myocardium we studied were not uniform between cases, because several pathologists performed and recorded the necropsies and chose the blocks. There is a strong chance that cases with left ventricular hypertrophy have been put in the group with no conventional evidence of abnormality, but assessment and recording of this varied between pathologists and we chose an arbitrary, objective measure of cardiomegaly. ${ }^{7}$ Possibly, if the whole of the left ventricle were to be examined in all cases, the extent of C9 immunostaining would be even more widespread than we found.

As it was, we found that $85 \%$ of necropsy hearts had evidence of $\mathrm{C} 9$ deposition. This was expected to some degree in 80 of the 109 cases, in whom there was conventional evidence of myocardial abnormality or coronary artery disease, although microscopy on haematoxylin and eosin sections detected muscle necrosis in only 12 . In the other 29 with C9 immunostaining, orthodox examination did not show damage (groups B and C, table 3). If these findings are generally applicable, then unsuspected acute myocardial damage is common in hospital necropsy cases. In fact it is almost universal. The C9 immunostaining excluded superimposed acute damage in eight hearts with chronic abnormality (group D, table 3) and this technique is helpful in confirmation or exclusion of acute events in such hearts.

Another striking finding of this study was that nearly every patient had at least one clinical feature that could explain myocardial damage, even if they had no obvious pathological changes in the heart. The most common risk factor was hypoxia. This is a reflection of the adult population that has a necropsy in a teaching hospital, because the patients are likely to have severe disease or a complicated clinical course or major medical and surgical procedures. There were not enough clinical details on duration or severity of illnesses to determine why some of the patients with the risk factors had no C9 deposition, but increasing age, heart weight, and total numbers of risk factors and pathological findings were associated with increasing grade of C9 immunostaining (tables 2 and 3 ).

Another method of detecting acute myocardial damage at necropsy is assessment of contraction band necrosis. Quantification of this was described and compared with analysis of cardiac isoenzymes in blood obtained after death. ${ }^{9}$ The method was described as potentially useful in the investigation of deaths in people with coronary atherosclerosis who were without obvious myocardial infarction or coronary thrombosis. ${ }^{10}$ A study compared detection of coagulative necrosis, contraction band necrosis, and deposition of the terminal complement complex C5b-9 in necropsy hearts. ${ }^{11}$ This showed that C5b-9 deposition was more widespread than changes detected by conventional methods. There were areas of contraction band necrosis that were negative for C5b-9 and were probably caused by agonal or resuscitation effects. The findings of the study on C $5 b-9^{11}$ support our findings with an antibody to C9. ${ }^{6}$

Immunostaining for C9 appears a simple and practical method of detecting acute myocardial damage at necropsy. It seems better than conventional pathological techniques and more elaborate methods such as enzyme histochemistry and quantification of contraction band necrosis. Implications of our study are that the myocardium is easily damaged and that this may be relevant to the cause of death in many hospital patients who have a necropsy.

1 Engel AG, Biesecker G. Complement activation in muscle fiber necrosis: demonstration of the membrane attack complex of complement in necrotic fibers. Ann Neurol 1982;12:289-96.

2 Schafer H, Mathey D, Hugo F, et al. Deposition of the terminal C5b-9 complement complex in infarcted areas of human myocardium. F Immunol 1986;137:1945-9.

3 Hugo F, Hamdoch T, Mathey D, et al. Quantitative measurement of SC5b-9 and C 5b-9 (m) in infarcted areas of human myocardium. Clin Exp

4 Mathey D, Schofer J, Schafer HJ, et al. Early accumulation of the terminal complement-complex in the ischaemic myocardium after reperfusion. Eur Heart $\mathcal{7}$ 1994;15:418-

5 Vakeva A, Morgan BP, Tikkanen F, et al. Time course of complement activation and inhibitor expression after ischaemic injury of rat myocardium. Am f Pathol 1994;144: 1357-68.

6 Doran JP, Howie AJ, Townend JN, et al. Detection of myocardial infarction by immunohistological staining for $\mathrm{C} 9$ on formalin fixed, paraffin wax embedded sections. $\mathcal{F}$ Clin Pathol 1996;49:34-7.

7 Kayser K. Height and weight in human beings: autopsy report. Munich: Verlag fur angewandte Wissenschaften, 1987: 104-13.

8 Flint EJ. Phenazine methosulphate and the nitroblue tetrazolium macroreaction for recent myocardial infarction. F Clin Pathol 1984;37:477-8.

9 Hopster DJ, Milroy CM, Burns J, et al. Necropsy study of the association between sudden cardiac death, cardiac soenzymes and contraction band necrosis. 7 Clin Pathol 1996;49:403-6.

10 Virmani R, Farb A, Burke A. Contraction-band necrosis: new use for an old friend. Lancet 1996;347:1710-11.

11 Edston E, Kawa K. Immunohistochemical detection of early myocardial infarction. An evaluation of antibodies against the terminal complement complex (C5b-9). Int $\mathcal{f}$ Legal Med 1995;108:27-30. 